Military Technical College

Kobry El-Kobbah,

Cairo, Egypt

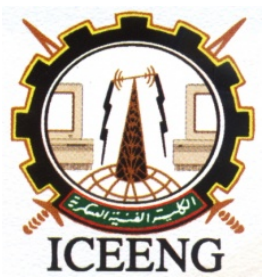

\section{1th International Conference \\ on Electrical Engineering}

ICEENG 2018

\title{
Design and Implementation of Emergency Response Notification for Distributed Control System
}

\author{
By \\ Eng. Omar Bekdash ${ }^{1}$, \\ Associate Prof.Alaa Hamdy ${ }^{2}$, Prof. Hassan El-Ghitany ${ }^{1}$ \\ ${ }^{1}$ Electronics and Communications Department, Faculty of Engineering, MIU \\ ${ }^{2}$ Faculty of Engineering, Helwan University
}

\begin{abstract}
Today's world is faced with many different types of emergencies in the industrial environment. Response to such emergencies is critical to protect resources including human life. In this work, an emergency response system is proposed which is easy to deploy and can report the emergency to the users in various forms, emergency response notification will be the preferred emergency systemin the future. A design foran emergency response system is presented using temperature,level, and pressure sensors as a proof of concept. The automated system achieves better performance than manual human response, it overcomes delayed informed danger, alarm priority and incorrectly estimating the type of danger or emergency.
\end{abstract}

\section{Keywords}

Emergency response, DCS, Notification, FM transceiver. 


\section{Introduction}

Working in industrialenvironment is very critical since it includes working in various types of hazardous areas(chemical vapors, fire,and explosion). In addition, siteswithpoor infrastructure may not be able to minimize loss of resources and human life in times of industrial catastrophes. The problem with most of the emergency response plan is the response itself, industrial catastrophes might happen in matter of seconds. So, reliable systems must be built to handle such situations with no place for human error (operator). The objective of this work is to limit incidents and prevent human loss due to human error. Most of industrial catastrophes can be prevented if the inform of the problem was done in fast and effective manner.

The objective of this work is to design integrated network to respond to any emergency and inform appropriate individuals in a timely and cost-effective manner. The research work further aims to enable ease of installations of variety of sensors and networking possibilities with a variety of networks such as Wireless Emergency Notification System (WENS) and Emergency Radio Notification Network (ERNN), to make sensor network and radio notification easily integrated with existing DCS systems.

The research work in [1] designed a system that responded to indoor emergencies as in universities. The research was concerned with emergencies that included gun violence and how students be informed and could be kept away from the source of danger and the appropriate authorities informed to deal with the source of danger. The research used cameras that were linked with sensor network that waslinked with university IT system and then system sends automated messages and notifications to the students and the authorities [1].

The paper is organized as follows; section II discusses the related work and the background of the research. Section III explains the main characteristics of the emergency system. Section IVdescribesthe proposed system, the theory of operationand the USRP and its hardware and software specifications. Section V obscures the experimental work and section VI comparesthe proposed system with other already built systems in the field.Finally, the conclusion is given in section VII.

Emergency systems recently have developed tremendously since the beginning of the 2000's but still many industrial catastrophes happened in the last decade due to human error. By human error we mean lack of information like the operator did not inform or explain the problem precisely or did not inform the appropriate team to deal with the problem like informing the maintenance team to deal with safety team job or the most common error which is the inform delay. Most of the industrial catastrophes would have been avoided if the inform was done properly and fast.

\section{Industrial catastrophes in the last decade}

These are some examples of industrial catastrophes in the last decade, e.g., the explosion in West Fertilizer Company inTexas at April 2013. In July 2013, another explosion happened in Geismar Plant, Louisiana. Also, Chevron Oil explosion occurred in Richmond California at August 2013. After that, Danlin Chemical Plant explosion that happened in Oklahoma at 
September 2013. Lately, in September 2016 a boiler explosion happened in Gazipur, Bangladesh lead to the death of 23 workers and the explosion was so powerful that the building of four floors collapsed [2].

The main properties of the emergency response notification system and its features.

\section{Emergency Radio Notification Network (ERNN)}

Most of industrial sites especially in petrochemical industry useradio phones for communication. So, the idea is to communicate with the appropriate teams in the site by sending automated recorded messages that are initiated from the emergency response system to the radio station through DCS to be delivered to the radio phones and the appropriate teams can act to such emergencies.

\section{Response time}

The response time in which the recorded message is delivered should be in matter of seconds which is much faster than normal operator response time, moreover it delivers the information more precisely thus the automated system is more reliable that manual systems [3].

\section{Characteristics of the emergency response system}

In this work, theexperimental work is having two USRP's one for transmission and the other for receiving, as shown in Figure 1. Two sensors;temperature and level; are used at the transmission front panel. In industrial applications, normally the sensor readings are compared certain thresholdsto detect the emergency cases. In this work, two thresholds are used.If the temperature sensor reading reaches $450{ }^{\circ} \mathrm{C}$ or the level sensor reading reaches $95 \%$, then a sound message will be sent for each case. to send the sound message, the sound message mustbe sampled and digitized first and then modulated by FM modulator.

In this work, USRP FM transceiver is used. The transmitted message from the first USRP FM transmitter has carrier frequency $97.5 \mathrm{MHZ}$ and I/Q rate 100k. Then, other USRP FM receiver receives the message, through this process it goesin FM demodulator then resampled with 10000 samples per secondto have a clear voice and then sound message is played.

In this work, if the two sensors reach the threshold points, the priority is set to the temperature sensor. So, its voice message is sent first and then other message of the level is sent second.

The most important factor which is response time as the sound message is transmitted, received, and played within less than 15 Seconds when the alarm is initiated. 


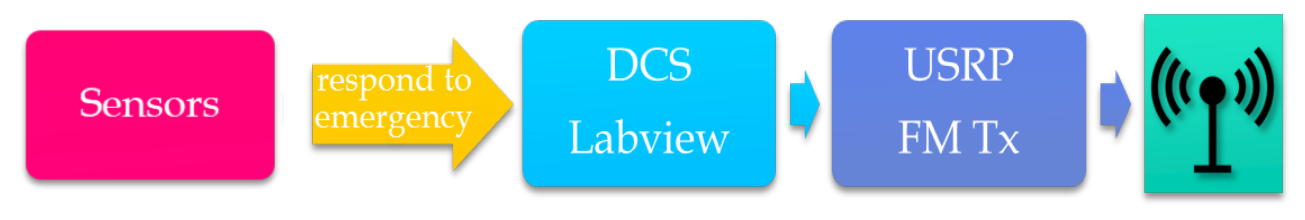

(a)

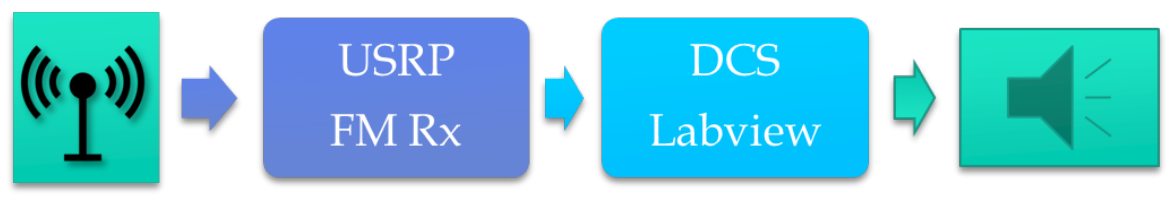

(b)

Fig. 1: The block diagram of the complete system; (a) Transmitter, (b) Receiver

\section{USRP}

Universal Software Radio Peripheral (USRP) is a range of software-defined radios designed and sold by Ettus Research and its parent company, National Instruments. Developed by a team led by Matt Ettus, the USRP product family is hardware platform for software radio, and is commonly used by research labs, universities [4].

Most USRPs connect to a host computer through a high-speed link, which the host-based software uses to control the USRP hardware and transmit/receive data. Some USRP models also integrate the general functionality of a host computer with an embedded processor that allows the USRP device to operate in a stand-alone fashion

The USRP family was designed for accessibility, and many of the products are open source hardware. The board schematics for select USRP models are freely available for download; all USRP products are controlled with the open source UHD driver, which is free and open source software. USRPs are commonly used with the GNU Radio software suite to create complex software-defined radio systems [5].

The USRP product family includes a variety of models that use a similar architecture. A motherboard provides the following subsystems: clock generation and synchronization, FPGA, ADCs, DACs, host processor interface, and power regulation. These are the basic components that are required for baseband processing of signals. A modular front-end, called a daughterboard, is used for analog operations such as up/down-conversion, filtering, and another signal conditioning. This modularity permits the USRP to serve applications that operate between DC and $6 \mathrm{GHz}$.

In stock configuration the FPGA performs several DSP operations, which ultimately provide translation from real signals in the analog domain to lower-rate, complex, baseband signals in the digital domain. In most use-cases, these complex samples are transferred to/from 
applications running on a host processor, which perform DSP operations. The code for the FPGA is open-source and can be modified to allow high-speed, low-latency operations to occur in the FPGA.

\section{NI USRPTM-29xx Hardware[6]}

The NI USRP connects to a host PC to act as a software-defined radio. Incoming signals attached to the standard SMA connector are mixed down using a direct-conversion receiver (DCR) to baseband I/Q components, which are sampled by a 2-channel, $100 \mathrm{MS} / \mathrm{s}, 14$-bit analog-to-digital converter (ADC), as shown in figure 2. The digitized I/Q data follows parallel paths through a digital down conversion (DDC) process that mixes, filters, and decimates the input $100 \mathrm{MS} / \mathrm{s}$ signal to a user-specified rate. The down converter samples, when represented as 32-bit numbers (16 bits each for I and Q), are passed to the host computer at up to $20 \mathrm{MS} / \mathrm{s}$ over a standard Gigabit Ethernet connection.

For transmission, baseband I/Q signal samples are synthesized by the host computer and fed to the USRP-2920 at up to $20 \mathrm{MS} / \mathrm{s}$ over Gigabit Ethernet when represented with 32-bits (16bits each for the I and Q components). The USRP hardware interpolates the incoming signal to $400 \mathrm{MS} / \mathrm{s}$ using a digital up conversion (DUC) process and then converts the signal to analog with a dual-channel, 16-bit digital-to-analog converter (DAC). The resulting analog signal is then mixed up to the specified carrier frequency.

An available 8-bit mode, in which 16-bits total are used to represent the I and Q values of a down converter sample or sample to be up converted, can enable a transfer rate of up to 40 $\mathrm{MS} / \mathrm{s}$ over the Gigabit Ethernet connection between the host PC and the USRP.

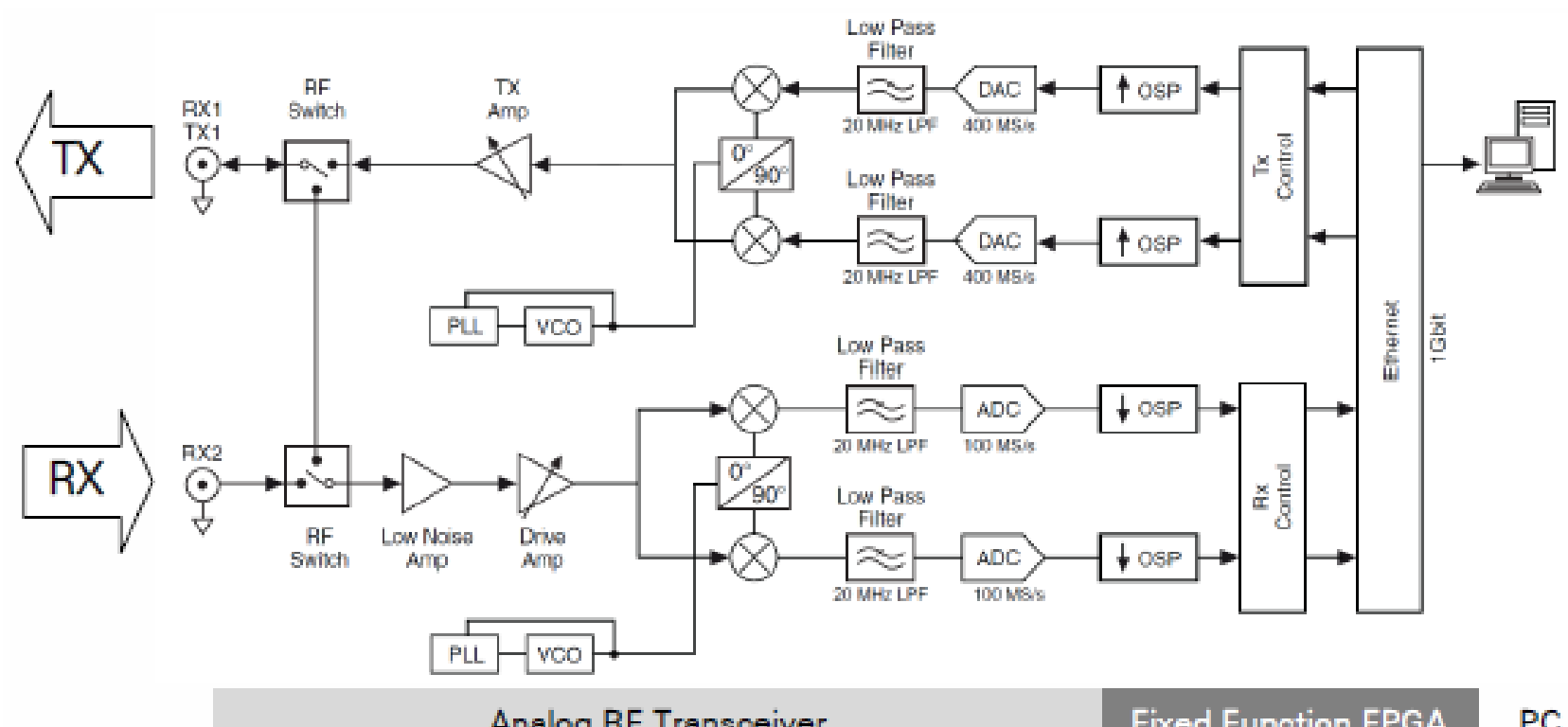

Fig. 2: NIUSRP Hardware 


\section{The proposed system}

In this section, the experimental work is presented. The figures below show the detailed work of this research. This section is divided into two parts; USRP FM receiver and USRP FM transmitter.

\section{A. USRP FM Receiver}

Figure 3 shows the FM receiver front panel and the overall block diagram is illustrated in figure 4(a). The detailed are demonstrated in figures $4(b, c, d)$.

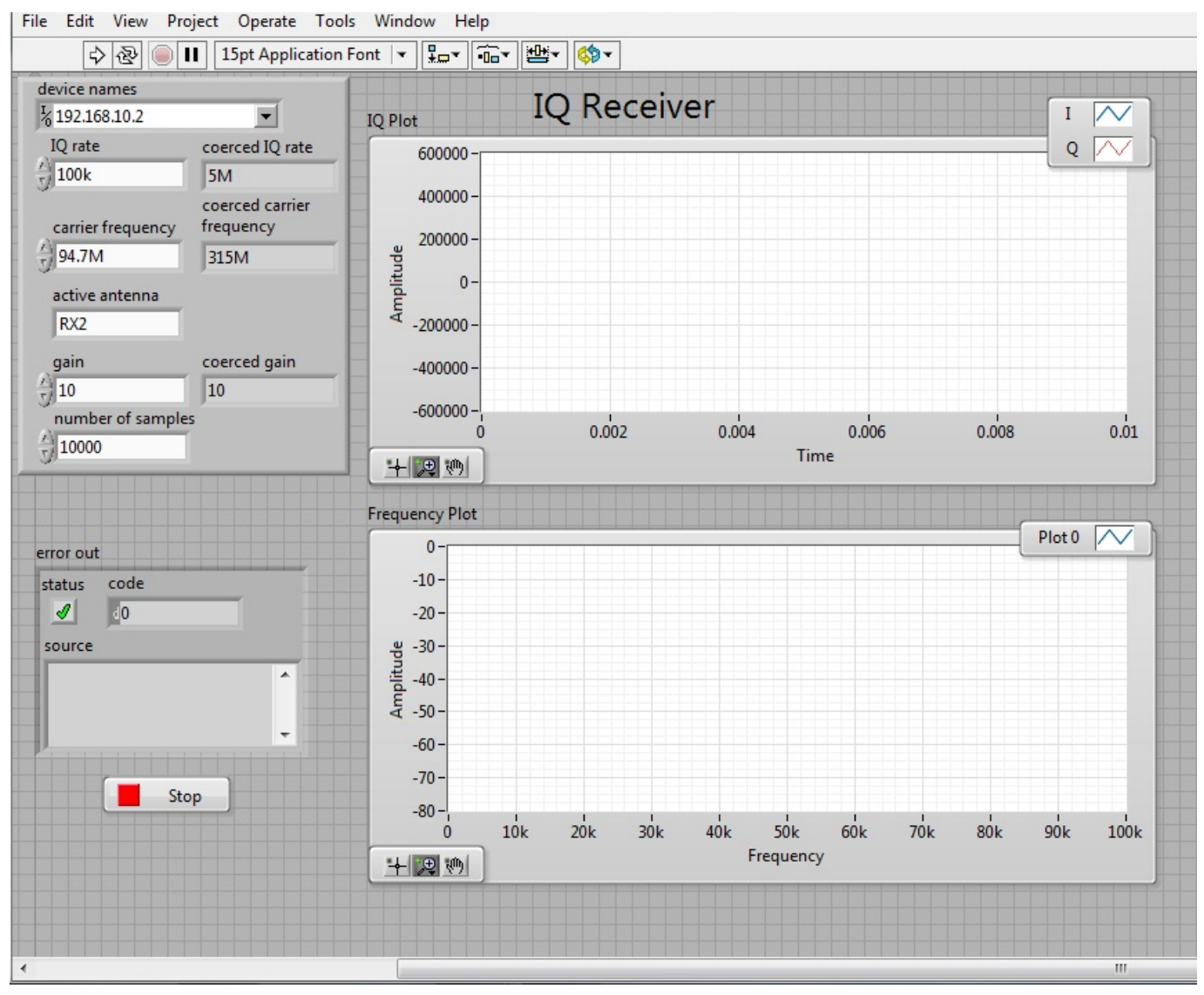

Fig.3: Receiver Front panel 


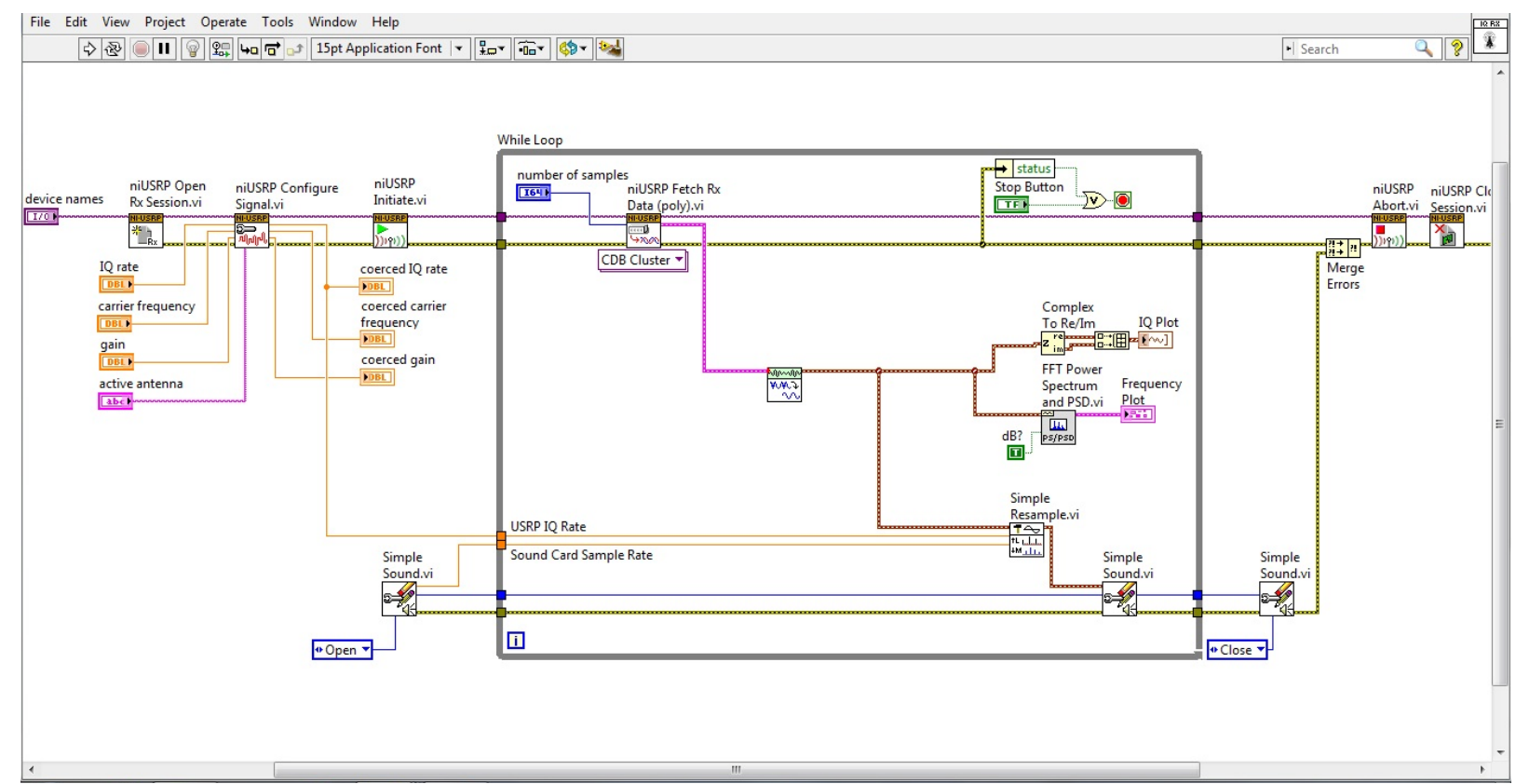

Fig.4(a): Receiver block diagram

Figure 4(a) is divided from left to right into three parts Figure 4(b, c, d), respectively and the devices are labeled by numbersto be explained in detail.

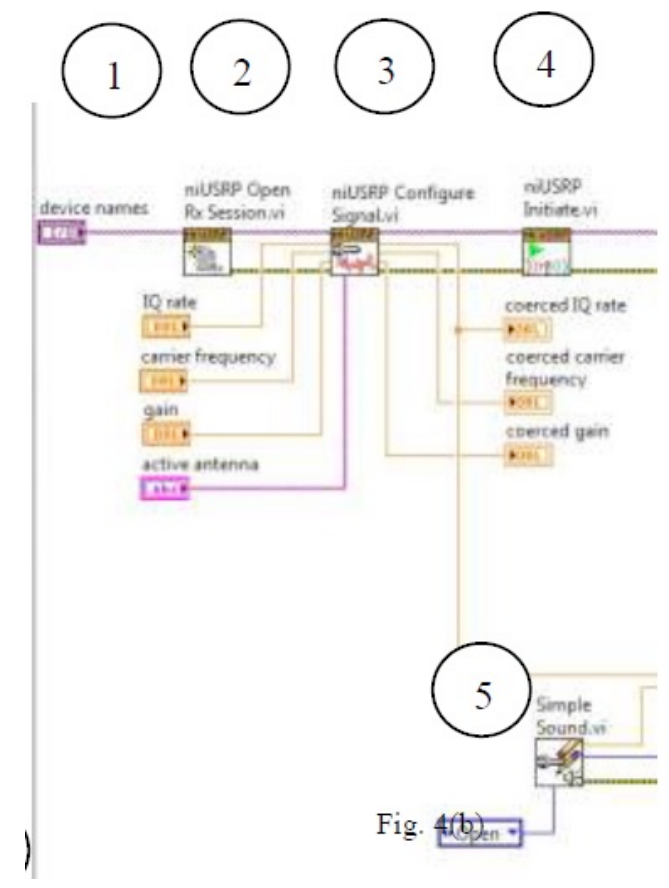

Fig. 4(b)

1. The device names terminal is linked to a front panel control and provides the IP address of the USRP 
2. niUSRP open Rx Session: creates a communication link between the program and the USRP. It returns a session handle on the purple wire.

3. niUSRP configure signal: sets the parameters for acquiring our signal. These parameters come from several different controls on the front panel. Actual parameters used by the USRP (coerced) get displayed by the front panel indicators.

4. niUSRP initiate: tells the USRP to start acquiring data.

5. Simple Sound: allows you to either open, write or close the sound card depending on the Action specified.

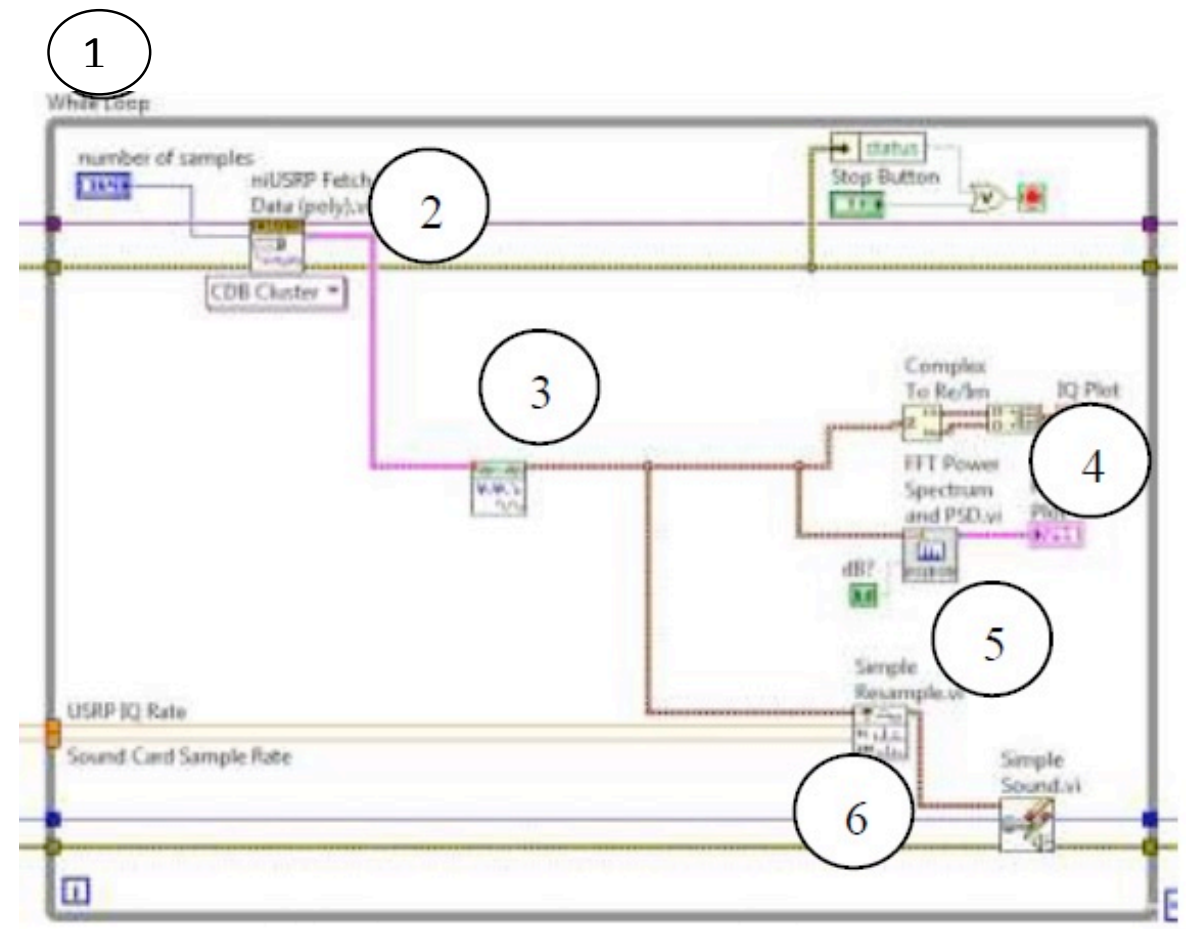

Fig. 4(c)

1. While loop to keep the FM receiver running for receiving FM signal

2. niUSRP fetch Rx data: Fetches Rx Data acquires a certain number of samples from the receive buffer.

3. FM demodulation and building Waveform function takes the dt value from the Fetch Rx

Data VI so the FFT plot and IQ plot scale correctly along the x-axis.

4. Graph I and Q components on a single plot.

5. Graph FFT of the signal.

6.Resamplethe demodulated data to $44.1 \mathrm{kHz}$ for the sound card. 


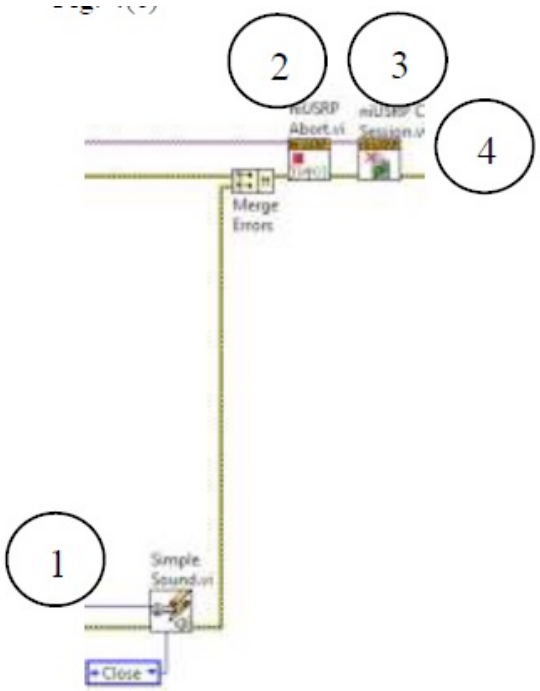

Fig. 4(d)

1. Simple Sound: closes the sound card so other programs can use it.

2. Merge errors: combines multiple error wires from parallel branches of code.

3.Abort: stops acquiring data and the Close Session VI frees any used memory.

4. Display any errors to the user on a front panel indicator.

\section{A. USRP FM Transmitter}

Figure 5 shows the FM transmitter front panel and the overall block diagram is illustrated in figure 6(a). The detailed are demonstrated in figures $6(b, c, d)$.

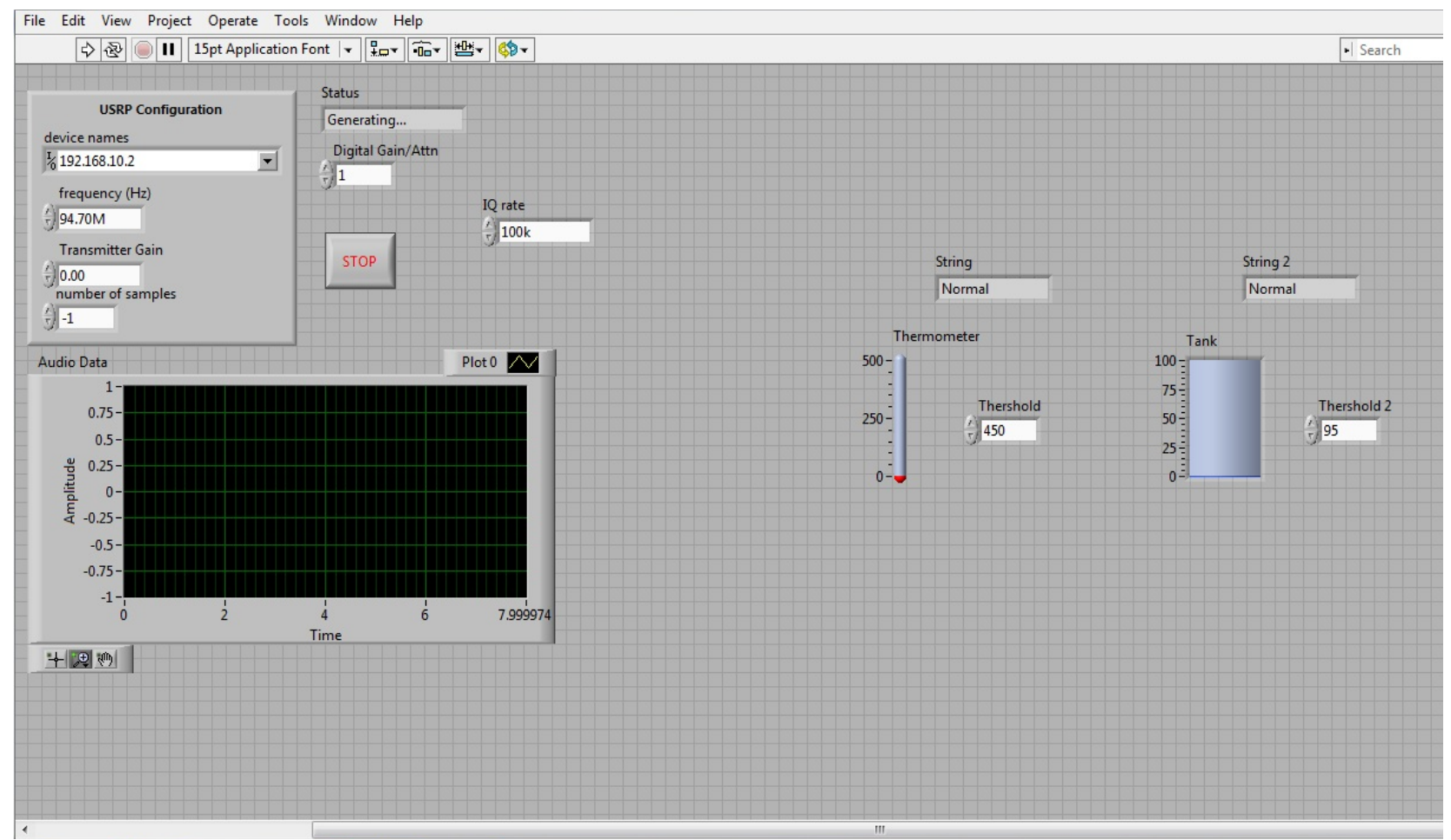

Fig.5: Transmitter Front panel 


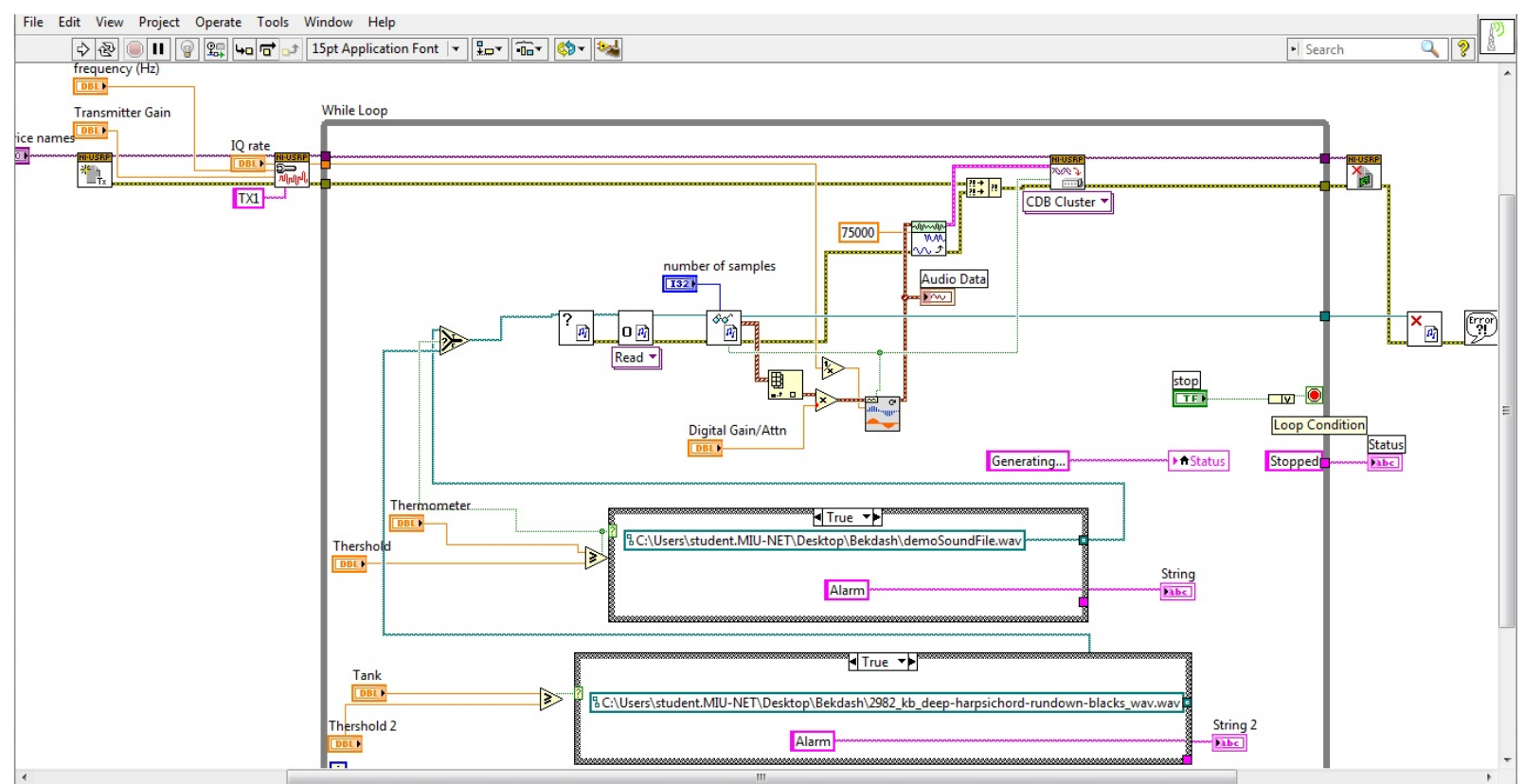

Fig.6(a): Transmitter block diagram

Figure 6(a) is divided from left to right into three parts Figure $6(\mathrm{~b}, \mathrm{c}, \mathrm{d})$, respectively and the devices are labeled by numbers to be explained in detail.

1. The device names terminal is linked to a front panel control and provides the IP address of the USRP

2. niUSRP Open Tx Session:creates a communication link between the program and the USRP. It returns a session handle on the purple wire.

3. Select the transmitting antenna

4. niUSRP Configure Signal:sets the parameters for transmitting our signal. These parameters come from several different controls on the front panel. Actual parameters used by the USRP (coerced) get displayed by the front panel indicators.

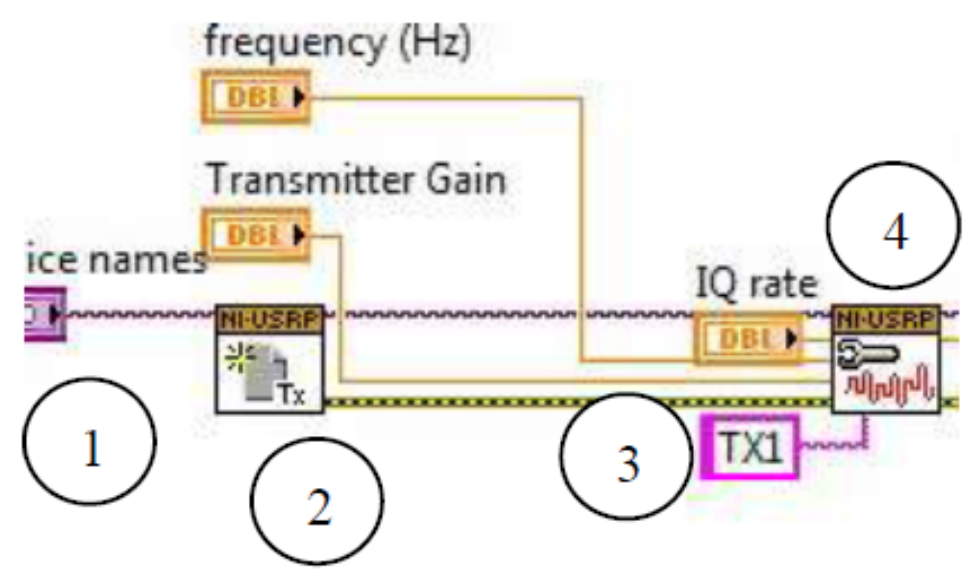

Fig.6(b) 


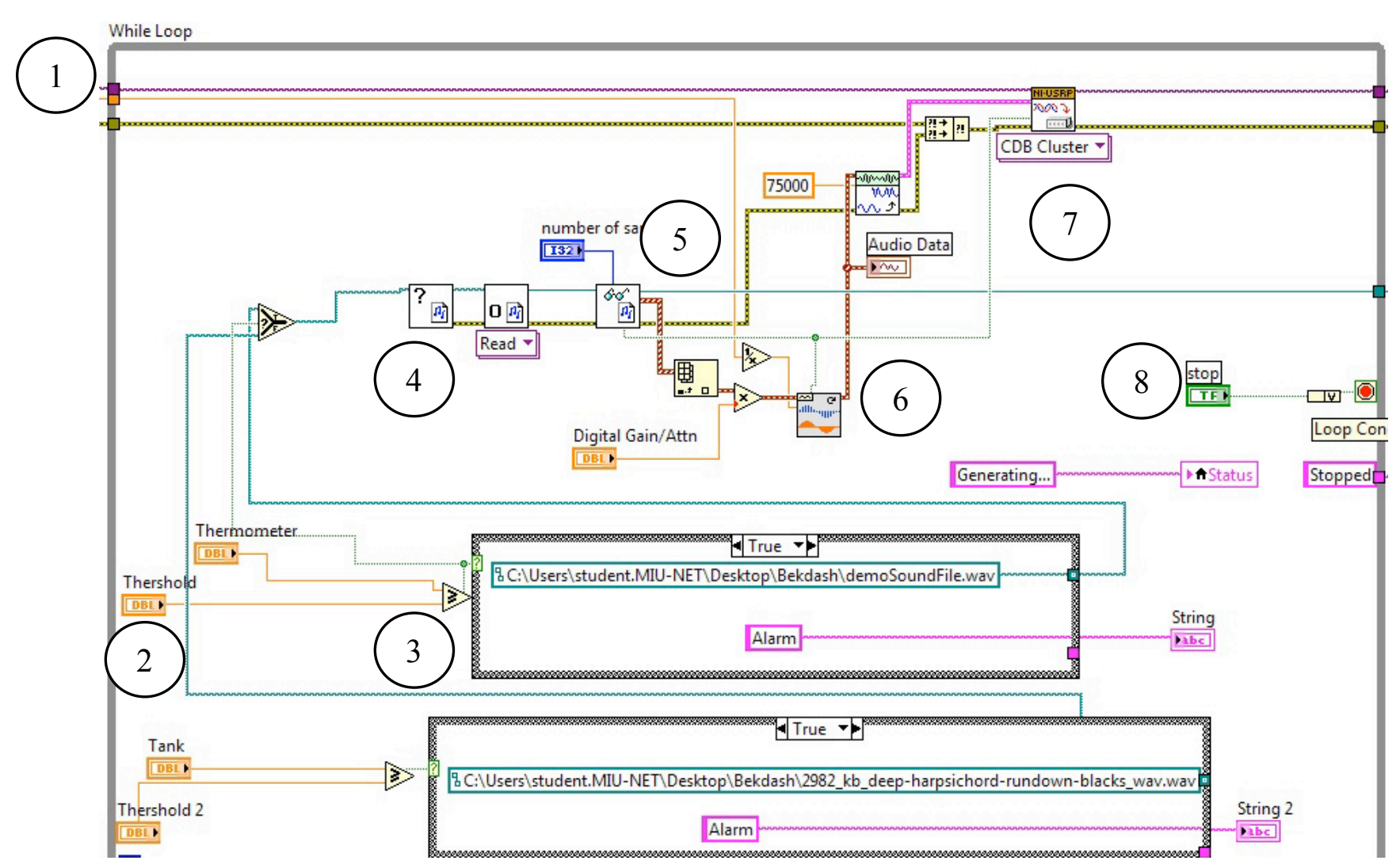

Fig.6(c)

1. While loop to keep the FM transmitter running continuously for any change in the sensors

2. We have 2 sensors Temperature sensor and level sensor with 2 threshold values when the sensor reading reaches the threshold value it sends the proper sound message that is collected with a collector

3. The sound files are put in if condition to be activated if the reading reaches the threshold

4. Reading the sound message file

5. Sampling digitizing is made for the sound file to prepare it to be send to the FM modulator

6. Resamples an input waveform according to the user-defined values for t0 and dt. Wire data to the waveform in input to determine the polymorphic instance to use or manually select the instance.

7. The Write Tx Data VI to send through the USRP

8. Stop button to stop the FM transmitter 

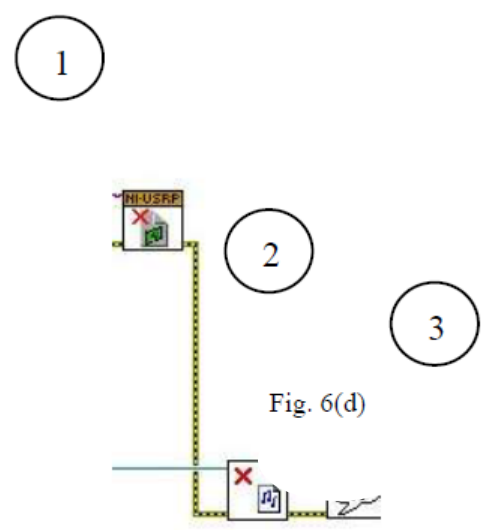

1. Close USRP session

Fig.6(d)

2. Close sound file

3. Collect the errors to be shown on the screen

\section{The Experimental testing}

The transmitted message from the first USRP FM transmitter has carrier frequency 97.5 MHZ and I/Q rate 100k. Then, other USRP FM receiver receives the message, through this process it goes in FM demodulator then resampled with 10000 samples per second to have a clear voice and then resampling is done tothe demodulated data to $44.1 \mathrm{kHz}$ for the sound card for playing the sound message.

\section{Conclusion}

This work presents an emergency response notification system. The proposed system is composed of FM transceiver, various types of sensors and a digital controller. When the sensor readings exceed the adjustable thresholds, a sound message is initiated consequently.

The system has the potential to significantly reduce the response time in a cost-effective way, as it is affordable and can be easily integrated with an existing DCS system. Moreover, it will limit the human errors and prevent industrial catastrophes and save human life.This system will be the preferred emergency system in the near future.

\section{References}

[1] A. Victor, A. Khader, C. Rao and A. Mehta "Build an IEEE 802.15.4 Wireless Sensor Network for Emergency Response Notification for Indoor Situations", based upon work supported by the National Science Foundation under Grant No. 0442313, published in IEEE 2011.

[2]Michael Cooney, "5 Chemical plant explosions", www.industrytap.com/5-chemical-plantexplosions-and-what-caused-them/1665,November20, 2013[Accessed:September10,2017]. 
[3]David A. Strobhar, Beville Engineering, and Craig M. Harvey, Louisiana State University, https://www.chemicalprocessing.com/articles/2011/how-many-alarms-can-an-operatorhandle/?start=1, 6 December 2011 [Accessed: September 10, 2017].

[4] Quinn Norton. "GNU Radio Opens an Unseen World". Wired.com. Retrieved 2014-04-18.

[5] "UHD Start". Ettus Research LLC. Retrieved 2012-09-05.

[6] An Introduction to Software Defined Radiowith NI LabVIEW and NI USRP Version 1.1 - Q4 2013.

[7] Karthikeyan s/o R. KrishnamurthyChairman, Workplace Safety and Health Council (Chemical Industries) Committee "WSHC Case Studies Chemical Industry", Published in June 2013 by the Workplace Safety and Health Council in collaboration with the Ministry of Manpower.

[8] Chetan S Kannur and Ramachandra. A. CInternational Journal of Engineering Research \& Technology (IJERT)ISSN: 2278-0181. Vol. 3 Issue 5, May - 2014 